\title{
Perfil do conhecimento de enfermeiros assistenciais sobre ações de prevenção e controle das infecções hospitalares
}

\author{
Knowledge profile of nursing assistants on actions to prevent and control \\ hospital infections
}

Perfil de los conocimientos de las enfermeras clínicas en la prevención y control de las infecciones hospitalarias

Juliana Aparecida Versiani de SOUZA ${ }^{(1)}$

Patrick Leonardo Nogueira da SILVA ${ }^{(2)}$

Renata Patrícia Fonseca GONÇALVES ${ }^{(3)}$

Recebido: 15 dez 2016

Revisado: 28 fev 2017

Aceito: 10 jan 2020

Autor de correspondência:

Patrick Leonardo Nogueira da Silva

patrick_mocesp70@hotmail.com

Conflito de interesses:

Os autores declaram não haver nenhum interesse profissional ou pessoal que possa gerar conflito de interesses em relação a este manuscrito.

\footnotetext{
${ }^{(1)}$ Faculdade de Medicina de Marabá - FACIMPA, Departamento de Medicina, Marabá, PA, Brasil. (2) Faculdades Unidas do Norte de Minas - FUNORTE, Departamento de Medicina, Montes Claros, MG, Brasil.

(3)Universidade Federal dos Vales do Jequitinhonha e Mucuri - UFVJM, Departamento de Enfermagem, Diamantina, MG, Brasil.
}

\section{Resumo}

A infecção hospitalar ocorre por meio da veiculação de microrganismos a um sítio de proliferação orgânico depois de 24 horas da internação hospitalar de um paciente de modo a gerar consequências patológicas e repercutir em um grave problema de saúde pública. $\mathrm{O}$ presente estudo objetivou identificar o perfil do conhecimento de enfermeiros assistenciais sobre ações de prevenção e controle de infecções hospitalares. Trata-se de um estudo descritivo, exploratório, com abordagem quantitativa, realizado com 23 enfermeiros assistenciais de um hospital universitário norte mineiro. Utilizou-se um questionário estruturado como instrumento de coleta de dados. O tratamento dos dados se deu por meio de epidemiologia descritiva com estatística não paramétrica. Observou-se prevalência de adultos jovens com idade entre 20-29 anos, do gênero feminino, apresentava pouca experiência no mercado de trabalho com tempo de atuação entre 0-4 anos. Quanto à participação em curso ou treinamento, 65,3\% participou. Destes, 33,4\% apresentava tempo de participação de 1-5 meses. Com relação à percepção sobre as infecções hospitalares, seus sítios de contaminação, as precauções padrão e suas vias de transmissibilidade, os participantes apresentaram uma média percentual de desconhecimento de 36,3\% tornando-se insatisfatória. Portanto, evidenciou-se um baixo nível de conhecimento entre os enfermeiros sendo necessária a programação de novos treinamentos, cursos e disponibilização de educação continuada de modo a contribuir na adoção de medidas de prevenção e controle de infecções hospitalares.

Descritores: Infecção Hospitalar; Precauções Universais; Enfermeiras e Enfermeiros; Conhecimento. 


\title{
Abstract
}

Hospital infection is an occurrence due to the transmission of microorganisms to a site of organic proliferation after 24 hours after the hospitalization of a patient in order to generate pathological consequences and repercussions in a serious public health problem. The present study aimed to identify the knowledge profile of nursing assistants on actions to prevent and control hospital infections. This is a descriptive, exploratory study with a quantitative approach, carried out with 23 nursing assistants from a university hospital in northern Minas Gerais. A structured questionnaire was used as instrument of data collection. The treatment of the data was done through descriptive epidemiology with non-parametric statistics. It was observed prevalence of young adults aged 20-29 years, female, with little experience in the labor market with a time of operation between 0-4 years. Regarding the participation in progress or training, $65.3 \%$ participated. Of these, $33.4 \%$ had participation time of 1-5 months. Regarding the perception about hospital infections, their contamination sites, standard precautions and their transmissibility pathways, the participants presented a mean percentage of unknowing of $36.3 \%$, becoming unsatisfactory. Therefore, a low level of knowledge among the nurses was evidenced, being necessary the programming of new training, courses and provision of continuing education in order to contribute to the adoption of measures of prevention and control of hospital infections.

Keywords: Cross Infection; Universal Precautions; Nurses; Knowledge.

\begin{abstract}
Resumen
La infección hospitalaria surge por medio del microorganismo que lleva a una proliferación orgánica de sitio de después de 24 horas de la hospitalización de un paciente con el fin de generar consecuencias patológicas y repercusiones en un grave problema de salud pública después de después de 24 horas de la hospitalización de un paciente con el fin de generar consecuencias patológicas y repercusiones en un grave problema de salud pública. Este estudio tuvo como objetivo identificar el perfil de los conocimientos del personal de enfermería clínica sobre prevención y control de las infecciones nosocomiales. Se trata de un estudio descriptivo, exploratorio con enfoque cuantitativo, realizado con 23 enfermeros clínicos de un hospital universitario del norte minero. Se utilizó un cuestionario estructurado como un instrumento de recolección de datos. El tratamiento de los datos fue por la epidemiología descriptiva con la estadística no paramétrica. Se observó que la mayoría de la muestra consistió en adultos jóvenes de 20-29 años, femenino, tenía poca experiencia en el mercado de trabajo con tiempo de funcionamiento comprendidas entre los $0-4$ años. En cuanto a la participación o la formación continua, 65,3\% participado. De éstos, 33,4\% mostró tiempo participación de 1-5 meses. En cuanto a la percepción de las infecciones nosocomiales, sus sitios de contaminación, las precauciones estándar y sus vías de transmisión, los participantes tuvieron un porcentaje promedio de $36,3 \%$ desconocimiento de llegar a ser insatisfactoria. Por lo tanto, se requiere evidencia de un bajo nivel de conocimiento entre las enfermeras para programar nuevos cursos de formación y prestación de la formación continua con el fin de contribuir a la adopción de medidas para prevenir las infecciones hospitalarias y de control.
\end{abstract}

Palabras-claves: Infección Hospitalaria; Precauciones Universales; Enfermeras y Enfermeros; Conocimiento.

\section{Introdução}

A infecção, de uma maneira genérica, é entendida como sendo uma ocorrência patológica que tem nos microrganismos a principal causa na qual independe do local ou do hospedeiro quando visto de forma geral. Com relação à infecção hospitalar (IH) ou nosocomial, a relação com o local muda de figura, pois, nesse caso, somente pacientes, depois da sua admissão em um hospital, estarão sujeitos a essa patologia. ${ }^{1}$ Todavia, não quer dizer que o paciente só contraia a doença no interior do hospital. Pode também ocorrer depois da sua saída (alta médica). 
O Ministério da Saúde - MS, por via da Portaria n. 2.616, de 12 de maio de 1998, ${ }^{2}$ considera que as IH constituem-se em significativo risco à saúde dos usuários de hospitais e, em contrapartida, que a prevenção e o controle envolvem medidas de qualificação da assistência hospitalar e de outros segmentos envolvidos. ${ }^{2} \mathrm{O}$ paciente, ao dar entrada em um hospital, necessariamente, estará dependente da assistência por parte dos profissionais de saúde envolvidos no seu processo terapêutico, incluindo-se, dentre esses profissionais, o enfermeiro. ${ }^{3}$ Não obstante, é missão de o enfermeiro assistencial prestar o atendimento, a assistência à saúde do indivíduo como um todo, até porque a enfermagem fundamenta-se no cuidar.

Em função da busca pela assistência à saúde, o paciente admitido no hospital fica à mercê tanto da instituição quanto dos profissionais. Porém, nem sempre essa assistência é praticada com a devida eficácia por diversas razões, tais como: omissão do trabalhador; sobrecarga por parte de outros funcionários; ou pela falta de conhecimento no qual não é incomum ocorrer em alguns hospitais. ${ }^{4} \mathrm{O}$ MS no Brasil expediu instruções de controle e prevenção das IH, por meio da Portaria n. 196, ${ }^{5}$ de 24 de junho de 1983, estabelecendo que todos os hospitais do país, independente da natureza da entidade mantenedora, deveriam ter Comissão de Controle de Infecção Hospitalar - $\mathrm{CCIH} .{ }^{6}$

Portanto, este estudo objetivou identificar o perfil do conhecimento de enfermeiros assistenciais sobre ações de prevenção e controle de IH.

\section{Metodologia}

Trata-se de um estudo descritivo, exploratório, com abordagem quantitativa, realizado no Hospital Universitário Clemente de Faria da Universidade Estadual de Montes Claros - HUCF/UNIMONTES, localizado na cidade de Montes Claros, Minas Gerais, Brasil. Foi enviada uma carta de apresentação e um Termo de Consentimento Institucional - TCI à Direção Clínica do HUCF/UNIMONTES, para autorização do estudo. A instituição foi devidamente orientada quanto às diretrizes da pesquisa e a mesma assinou o TCI de modo a autorizar a realização da pesquisa.

O universo de profissionais enfermeiros existentes na instituição em estudo é de 85 enfermeiros, entretanto, a amostra pesquisada foi de 23 profissionais, representando $27 \%$ do universo, considerando essa amostra não probabilística por acessibilidade, ou seja, os elementos pesquisados foram selecionados segundo a disponibilidade no local e no momento em que a coleta de dados estava sendo realizada. ${ }^{7}$ 
O recrutamento dos sujeitos da pesquisa foi realizado a partir de carta convite, ressaltando-se que a participação foi voluntária e que suas identidades foram preservadas. $\mathrm{O}$ período de pesquisa foi entre os meses de fevereiro a junho de 2008.

Foi adotado o seguinte critério de inclusão para participação no estudo: ser enfermeiro com atuação assistencial. Quanto aos critérios de exclusão, tem-se: enfermeiros ausentes em virtude de atestado médico; enfermeiros em caráter de licença e/ou afastamento; enfermeiros que estiveram de férias.

Foi utilizado um questionário estruturado como instrumento de coleta de dados direcionado aos participantes da pesquisa. Realizou-se um pré-teste do instrumento de coleta de dados com enfermeiros de outra instituição de saúde para identificar as necessidades de revisão e correção. O questionário contemplou assertivas de acordo com as seguintes categorias: perfil epidemiológico; conhecimento sobre prevenção e controle das $\mathrm{IH}$; e conhecimento sobre precaução das IH. A análise estatística foi realizada por meio do programa Microsoft Excel ${ }^{\circledR}$ 7.0, versão 2003, na qual os dados foram apresentados em tabelas e expressos as frequências absolutas (n) e percentuais (\%). O tratamento dos dados se deu através de epidemiologia descritiva.

Este estudo respeitou os preceitos éticos estabelecidos pela Resolução n. $196^{8}$, de 10 de outubro de 1996, do Conselho Nacional de Saúde - CNS, no qual regulamenta a realização de pesquisa envolvendo seres humanos. O projeto de pesquisa foi apreciado e aprovado pelo Comitê de Ética em Pesquisa da Universidade Estadual de Montes Claros - CEP/UNIMONTES sob parecer consubstanciado n. 1029/2008. Os participantes foram orientados quanto às diretrizes do estudo e assinaram o Termo de Consentimento Livre e Esclarecido - TCLE autorizando a participação voluntária no estudo.

\section{Resultados}

Observou-se prevalência de enfermeiros assistenciais eram adultos jovens com idade entre 20-29 anos (56,5\%), do gênero feminino (65,3\%), com tempo de atuação entre 0-4 anos (56,5\%). Quanto à participação em curso ou treinamento, 65,3\% participou. Destes, 33,4\% apresentava tempo de participação de 1-5 meses (Tabela 1).

Tabela 1. Perfil epidemiológico dos enfermeiros assistenciais do HUCF/UNIMONTES. Montes Claros (MG), 2008.

\begin{tabular}{|l|c|c|c|}
\hline \hline \multicolumn{1}{|c|}{ Variável } & Descrição & $\mathrm{n}=23$ & $\%$ \\
Idade & De 20 a 29 anos & 13 & 56,5 \\
& De 30 a 39 anos & 08 & 34,7 \\
& De 40 a 49 anos & 02 & 8,8 \\
& De 50 a 59 anos & 00 & 00 \\
& Acima de 60 anos & 00 & 00
\end{tabular}


ISSN 2179-6750

\begin{tabular}{l|c|c|c}
\hline \multicolumn{1}{c|}{ Variável } & Descrição & $\mathrm{n}=23$ & $\%$ \\
\hline \multirow{3}{*}{ Gênero } & Masculino & 08 & 34,7 \\
& Feminino & 15 & 65,3 \\
& De zero a 04 anos & 13 & 56,5 \\
& De 05 a 09 anos & 07 & 30,4 \\
& De 10 a 14 anos & 01 & 4,3 \\
Participação em & De 15 a 19 anos & 02 & 8,8 \\
curso ou treinamento & Acima de 20 anos & 00 & 00 \\
Tempo de & Sim & 15 & 65,3 \\
participação (n=15) & Não & 08 & 34,7 \\
& De 01 a 05 meses & 05 & 33,4 \\
& De 06 a 12 meses & 04 & 26,6 \\
& Acima de 12 meses & 03 & 20,0 \\
\hline \hline
\end{tabular}

Fonte: Elaborado pelos autores (2008).

Os participantes foram unanimes em descrever a IH como qualquer infecção adquirida após a internação do paciente e que se manifesta durante a internação ou após a alta, quando puder ser relacionada à internação ou com os procedimentos hospitalares. Observou-se que $65,2 \%$ considera incorreta a periodicidade da troca da sonda vesical de demora (SVD), sendo esta de 15 em 15 dias. Quanto à infecção por pneumonia (PNM), considerou-se incorreto por 69,5\% que a manutenção do umidificador da oxigenoterapia não invasiva deve ser realizada com água estéril de modo a facilitar o trabalho em situações de emergência. Em se tratando da infecção do sítio cirúrgico (ISC), é incorreto afirmar, de acordo 52,1\% dos enfermeiros, que a principal fonte de infecção são os instrumentos utilizados no ato da cirurgia. O curativo do cateter venoso central (CVC) deve ser trocado a cada 72 horas e pode ser feito por qualquer profissional de enfermagem caracteriza-se uma assertiva falsa para $48 \%$ da amostra quando relacionado às infecções por cateter vascular (ICV) (Tabela 2).

Tabela 2. Perfil do conhecimento dos enfermeiros assistenciais acerca das IH e seus sítios de contaminação. Montes Claros (MG), 2008.

\begin{tabular}{lcc}
\hline \hline \multicolumn{1}{c}{ Variáveis } & $\mathrm{n}=23$ & $\%$ \\
\hline $\begin{array}{l}\text { Afirmação correta sobre IH } \\
\text { IH infecções transmitidas por via placentária são consideradas }\end{array}$ & 00 & 00 \\
& 00 & 00 \\
& & \\
IH é a infecção constatada ou em incubação no ato da & $\begin{array}{l}\text { admissão do paciente, desde que não relacionada com } \\
\text { internação anterior no mesmo hospital; }\end{array}$ & 100 \\
IH é qualquer infecção adquirida após a internação do \\
$\begin{array}{l}\text { paciente e que se manifesta durante a internação ou após a } \\
\text { alta, quando puder ser relacionada à internação ou com os } \\
\text { procedimentos hospitalares; }\end{array}$
\end{tabular}


ISSN 2179-6750

\begin{tabular}{|c|c|c|}
\hline Variáveis & $n=23$ & $\%$ \\
\hline $\begin{array}{l}\text { São hospitalares as infecções associadas à bolsa rota superior } \\
\text { a } 24 \text { horas. }\end{array}$ & 00 & 00 \\
\hline \multicolumn{3}{|l|}{ Afirmação incorreta sobre infecção do trato urinário (ITU) } \\
\hline A troca da sonda de demora deve ser feita a cada 15 dias; & 15 & 65,2 \\
\hline $\begin{array}{l}\text { As indicações para troca de sonda vesical de demora são: } \\
\text { febre de origem indeterminada, obstrução do sistema coletor } \\
\text { e piúria; }\end{array}$ & 03 & 13 \\
\hline $\begin{array}{l}\text { Dentre as indicações para passagem de sonda vesical de } \\
\text { demora pode-se citar: paciente com bexiga neurogênica, para } \\
\text { medir débito urinário rigoroso e pós-operatório de cirurgias } \\
\text { do trato urinário; }\end{array}$ & 03 & 13 \\
\hline $\begin{array}{l}\text { Não se deve deixar o volume urinário ultrapassar } 2 / 3 \text { da } \\
\text { capacidade da bolsa coletora. }\end{array}$ & 02 & 8,8 \\
\hline \multicolumn{3}{|l|}{ Afirmação incorreta sobre infecção por PNM } \\
\hline $\begin{array}{l}\text { Os maiores índices de PNM em paciente submetido à } \\
\text { ventilação mecânica prolongada; }\end{array}$ & 00 & 00 \\
\hline $\begin{array}{l}\text { O risco aumentado de desenvolver PNM em paciente } \\
\text { submetido à intubação endotraqueal deve-se entre outras } \\
\text { causas, ao transporte direto de organismos da orofaringe } \\
\text { através do tubo endotraqueal durante a realização do } \\
\text { procedimento; }\end{array}$ & 06 & 26,2 \\
\hline $\begin{array}{l}\text { O tubo é corpo estranho que traumatiza frequentemente o } \\
\text { epitélio, favorecendo a aderência bacteriana e colonização } \\
\text { das vias aéreas, atuando como reservatório de proliferação } \\
\text { microbiana; }\end{array}$ & 01 & 4,3 \\
\hline $\begin{array}{l}\text { O umidificador da oxigenoterapia não invasiva deve sempre } \\
\text { ser mantido com água estéril para facilitar o trabalho nas } \\
\text { situações de emergência. }\end{array}$ & 16 & 69,5 \\
\hline \multicolumn{3}{|l|}{ Afirmação incorreta sobre ISC } \\
\hline $\begin{array}{l}\text { A principal fonte de infecção são os instrumentos utilizados } \\
\text { no ato da cirurgia; }\end{array}$ & 12 & 52,1 \\
\hline $\begin{array}{l}\text { O momento do ato cirúrgico é o período crítico para } \\
\text { contaminação bacteriana da ferida operatória; }\end{array}$ & 02 & 8,6 \\
\hline $\begin{array}{l}\text { É considerada ISC, infecções acometidas em até um ano se } \\
\text { houver implantação de material protético; }\end{array}$ & 07 & 30,7 \\
\hline $\begin{array}{l}\text { Fatores de risco para infecções de sítio cirúrgico incluem } \\
\text { diabetes mellitus (DM), tabagismo, desnutrição e idade } \\
\text { avançada. }\end{array}$ & 02 & 8,6 \\
\hline \multicolumn{3}{|l|}{ Afirmação incorreta sobre ICV } \\
\hline $\begin{array}{l}\text { O índice de complicações infecciosas associadas aos acessos } \\
\text { vasculares varia de acordo com o tipo de cateter e a } \\
\text { freqüência de manipulação dos mesmos; }\end{array}$ & 03 & 13 \\
\hline $\begin{array}{l}\text { A maioria das infecções decorre de microrganismos que } \\
\text { colonizam as conexões da pele adjacentes ao sítio de inserção } \\
\text { do cateter; }\end{array}$ & 03 & 13 \\
\hline $\begin{array}{l}\text { Deve-se trocar o cateter venoso em adulto a cada } 96 \text { horas } \\
\text { com o intuito de prevenir infecção; }\end{array}$ & 06 & 26 \\
\hline
\end{tabular}


ISSN 2179-6750

\begin{tabular}{ccc}
\hline Variáveis & $\mathrm{n}=23$ & $\%$ \\
\hline O curativo do CVC deve ser trocado a cada 72 horas e pode & 11 & 48 \\
ser feito por qualquer profissional de enfermagem. & & \\
\hline
\end{tabular}

Fonte: Elaborado pelos autores (2008).

Na concepção de $82,8 \%$ dos entrevistados, as precauções padrão são medidas de higienização das mãos na qual contemplam o uso de luvas para o contato com todos os pacientes internados. Foi considerada uma alegação incorreta por 47,9\% que as precauções baseadas na transmissão são designadas apenas para o cuidado de pacientes conhecidos ou suspeitos de estarem infectados. Em caso de tuberculose pulmonar, a precaução utilizada é por aerossóis (73,9\%). Neste tipo de precaução, os Equipamentos de Proteção Individual - EPI e/ou medidas preventivas que devem ser utilizados pela equipe são: uso de avental; máscara N-95; higienização das mãos; e alocação do paciente em quarto privativo obrigatório/coorte/porta fechada (Tabela 3).

Tabela 3. Perfil do conhecimento dos enfermeiros assistenciais acerca das precauções padrões e suas vias de transmissibilidade. Montes Claros (MG), 2008.

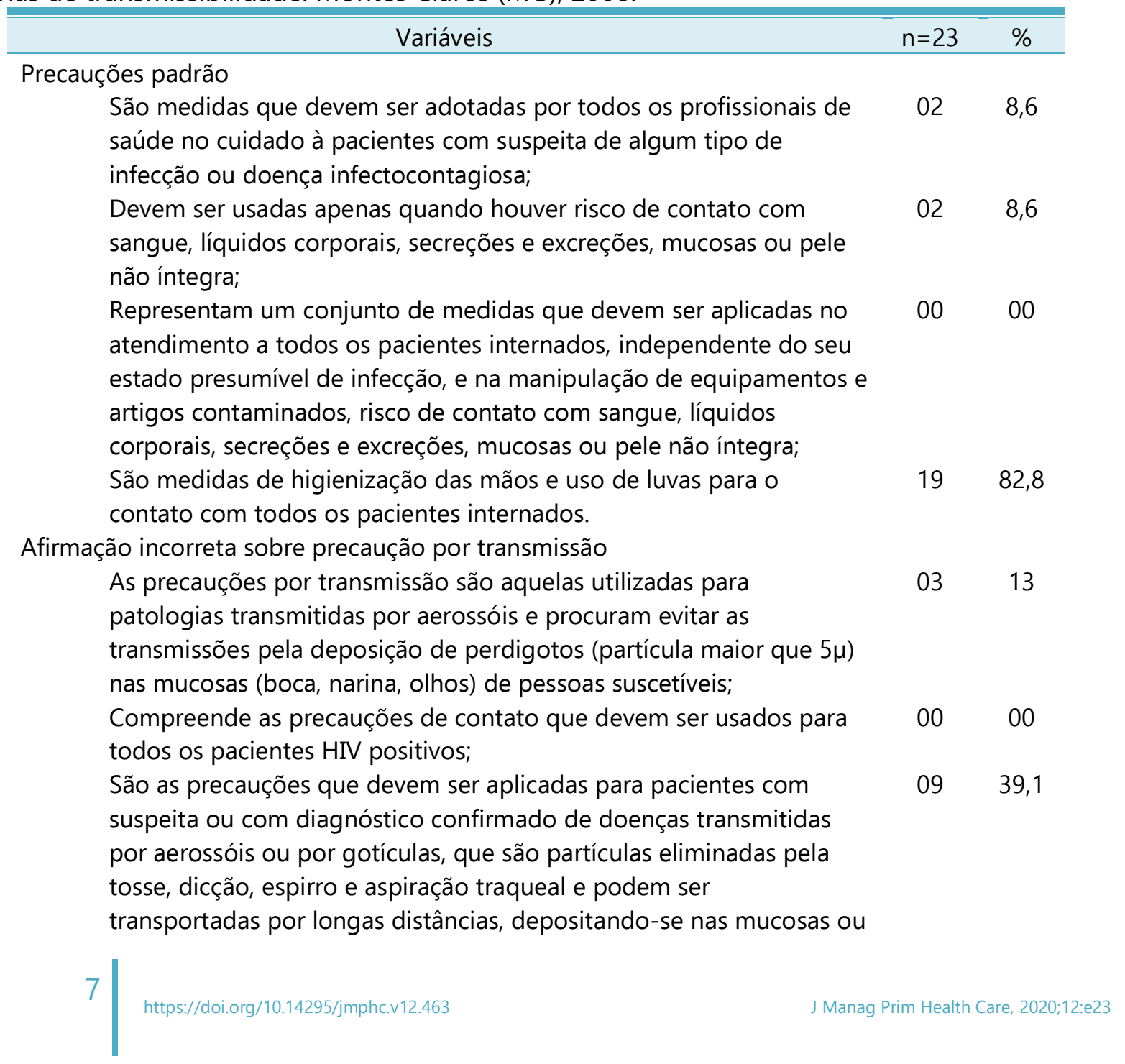


ISSN 2179-6750

\begin{tabular}{|c|c|c|c|}
\hline \multicolumn{2}{|l|}{ Variáveis } & $\mathrm{n}=23$ & $\%$ \\
\hline \multicolumn{4}{|c|}{$\begin{array}{l}\text { sendo inaladas por pessoas suscetíveis, principalmente, quando } \\
\text { houver correntes de ar; }\end{array}$} \\
\hline \multicolumn{2}{|c|}{$\begin{array}{l}\text { As precauções baseadas na transmissão são designadas apenas para } \\
\text { o cuidado de pacientes conhecidos ou suspeitos de estarem } \\
\text { infectados ou colonizados por microrganismos importantes } \\
\text { epidemiologicamente e que podem ser transmitidos através de } \\
\text { aerossóis, gotículas ou por contato. }\end{array}$} & 11 & 47,9 \\
\hline \multicolumn{4}{|l|}{ Precaução em caso de tuberculose pulmonar } \\
\hline \multicolumn{2}{|l|}{ Precaução por contato; } & 00 & 00 \\
\hline \multicolumn{2}{|l|}{ Precaução por aerossóis; } & 17 & 73,9 \\
\hline \multicolumn{2}{|l|}{ Precaução por gotícula; } & 06 & 26,1 \\
\hline \multicolumn{2}{|l|}{ Nenhuma precaução. } & 00 & 00 \\
\hline \multicolumn{4}{|c|}{ Precaução em caso de doenças transmitidas por aerossóis } \\
\hline \multirow[t]{2}{*}{ Máscara cirúrgica } & Sim & 05 & 21,7 \\
\hline & Não & 18 & 78,3 \\
\hline \multirow[t]{2}{*}{ Avental } & Sim & 16 & 69,5 \\
\hline & Não & 07 & 30,5 \\
\hline \multirow[t]{2}{*}{ Luvas } & Sim & 11 & 47,8 \\
\hline & Não & 12 & 52,2 \\
\hline \multirow[t]{2}{*}{ Máscara N-95 } & Sim & 21 & 91,3 \\
\hline & Não & 02 & 8,7 \\
\hline \multirow[t]{2}{*}{ Higienização das mãos } & Sim & 16 & 69,5 \\
\hline & Não & 07 & 30,5 \\
\hline \multirow[t]{2}{*}{ Óculos } & Sim & 09 & 39,1 \\
\hline & Não & 14 & 60,9 \\
\hline \multirow[t]{2}{*}{ Quarto privativo/coorte } & Sim & 10 & 43,4 \\
\hline & Não & 13 & 56,6 \\
\hline \multirow[t]{2}{*}{ Quarto privativo obrigatório/coorte/porta fechada } & Sim & 14 & 60,9 \\
\hline & Não & 09 & 39,1 \\
\hline
\end{tabular}

Fonte: Elaborado pelos autores (2008).

\section{Discussão}

Estudo enfatiza que os trabalhadores da enfermagem são relativamente jovens e quanto menor a idade, menor é a oportunidade de seu preparo profissional. ${ }^{9}$ Dentro da própria área, há um predomínio feminino. ${ }^{10}$ Os cursos de aperfeiçoamento e treinamento preparam o profissional para atuar na prática e evitar erros advindos de despreparo técnicocientífico. Sendo assim, a diminuição no investimento intelectual propicia a disseminação de IH no ambiente profissional. ${ }^{11}$ Neste estudo, o corpo de enfermagem do HUCF pode ser classificado como sendo predominantemente feminino e composto por profissionais jovens. O resultado obtido em relação à participação dos pesquisados em cursos e treinamento, mostra que, embora $65,3 \%$ já tenham participado de cursos e treinamentos, é significativo o índice dos que nunca participaram. O que não deixa de ser uma questão preocupante, 
visto que $56,5 \%$ dos profissionais de enfermagem é jovem. Os achados na literatura científica corroboram com os dados deste estudo.

Na questão relacionada ao entendimento sobre $\mathrm{IH}$, o resultado foi unânime. Todos afirmaram que IH é qualquer infecção adquirida após a internação do paciente e que se manifesta durante a mesma ou após a alta, quando puder ser relacionada à internação ou com os procedimentos hospitalares. Tais resultados vêm afirmar que o conhecimento dos pesquisados sobre $\mathrm{IH}$ foi bastante positivo, o que pode ser ratificado pelo entendimento de um estudioso na qual a IH é uma infecção que tem como principal causa os microrganismos e ainda, que independe do local ou do hospedeiro, quando visto de forma geral, observando que nos casos nosocomiais, a relação com o local muda de figura, pois, nesse caso, somente pacientes, depois da sua admissão em um hospital, estará sujeito a essa patologia. ${ }^{1}$

Como se pode observar, em conformidade com o manual de prevenção e controle das IH do HUCF, ${ }^{12}$ percebe-se que $65,2 \%$ dos profissionais dominam o conhecimento em relação às prevenções de ITU, uma vez que estes consideraram, acertadamente, que é incorreto afirmar que a troca da sonda de demora deve ser feita a cada 15 dias. É orientado por outros estudos que essa troca pode expor o paciente ao risco de infecções nosocomiais. ${ }^{13,14}$ No manual de prevenção e controle das IH do HUCF, ${ }^{12}$ bem como em outras publicações, ${ }^{15,16}$ é incorreto afirmar que o umidificador da oxigenoterapia não invasiva deve sempre ser mantido com água estéril para facilitar o trabalho nas situações de emergências. Neste estudo, esse resultado leva a interpretar que menos da metade responderam acertadamente, levando ao entendimento de ser baixo o conhecimento do corpo de enfermagem do HUCF em relação às PNM hospitalares.

Os resultados mostram que, em relação à prerrogativa incorreta sobre ISC, mais da metade dos enfermeiros assistenciais pesquisados optaram por considerar incorreto o fato de que a principal fonte de infecção são os instrumentos utilizados no ato da cirurgia, quando na realidade são os próprios pacientes, por questões endógenas do tipo: infecções preexistentes, idade elevada, riscos anestésicos, DM, neoplasia, desnutrição, tabagismo, dentre outros. ${ }^{13,17}$ Analogamente à ISC, 48\% da amostra salientam ser incorreto afirmar que o curativo do CVC deve ser trocado a cada 72 horas podendo ser feito por qualquer profissional de enfermagem quando se tratado da ICV. É importante destacar que a periodicidade de troca para cobertura com gaze consiste em 24 horas a 48 horas e para filme transparente até no máximo sete dias ou quando apresentar sujidade, umidade, enrugamento, soltura ou algum outro comprometimento. ${ }^{18,19}$

Quanto às precauções padrão, evidencia-se que $82,8 \%$ dos profissionais participantes dessa pesquisa entendem satisfatoriamente a sua definição. Nas percepções sobre precauções por transmissão, novamente a inconsistência de entendimento se faz presente, 
pois 47,9\% entendem de maneira correta que as precauções baseadas na transmissão são designadas apenas para o cuidado de pacientes conhecidos ou suspeitos de estarem infectados ou colonizados por microrganismos importantes epidemiologicamente e que podem ser transmitidas através de aerossóis, gotículas ou por contato. ${ }^{20}$ Assim, no que concerne aos tipos de precauções orientadas para a tuberculose pulmonar, verificou-se que 73,9\% entende corretamente que o tipo característico é a precaução por aerossóis. ${ }^{21}$ Entretanto, mais de $25 \%$ destes entendem ser a precaução por gotículas, retomando a questão de preocupação com o conhecimento dos profissionais assistenciais.

Em outra pesquisa é afirmada que esta infecção se deve a interações ambientais. $^{22}$ Conforme o Hospital Universitário Clementino Fraga Filho da Universidade Federal do Rio de Janeiro - HUCFF/UFRJ em relação à precaução recomenda, cuidados nas transmissões pelo ar, sendo obrigatório o uso de máscara, de quarto privativo e de máscara cirúrgica nos transportes de pacientes, fica evidente que a precaução mais indicada é a precaução por aerossóis. ${ }^{23}$ Em se tratando de utilização de EPI e medidas preventivas com relação à infecção por aerossóis, tem-se: a utilização de quarto privativo/coorte com portas fechadas; uso obrigatório de máscara tipo N-95 e; evitar o transporte do paciente, mas quando necessário o paciente deve estar utilizando máscara cirúrgica comum. ${ }^{24}$ Entretanto, observou-se dissonância entre as percepções dos enfermeiros respondentes, que pontuaram em índices significativos: avental, luvas, óculos e, em quantidades menores da esperada, a utilização de máscara cirúrgica.

A média percentual do conhecimento, na teoria da probabilidade e estatística, significa a medida da sua dispersão estatística, indicando o quão longe os valores encontrados estão do valor esperado. Daí o fato de se preocupar, pois, considerando que entre a relação do conhecimento dos enfermeiros assistentes e da eficácia das suas ações estão as vidas dos pacientes por eles atendidos. Com isso, cabe sugerir que sejam efetivados novos cursos e treinamentos, no sentido de prepará-los melhor em relação ao conhecimento das $\mathrm{IH}$, bem como das suas devidas precauções.

\section{Conclusão}

Evidenciou-se um baixo nível de conhecimento entre os participantes, uma vez que a média foi de 36,3\% na qual representa um índice de alta significância com vistas pela instituição a preocupar-se no investimento de medidas de aperfeiçoamento dos mesmos. Os resultados apontaram que o conhecimento dos profissionais pesquisados em relação a medidas de precaução da transmissão de doenças infectocontagiosas não pode ser dado como satisfatório. Portanto, em virtude dos resultados, sugere-se ser necessária a 
implementação de novos treinamentos, cursos e disponibilização de educação continuada, para os entrevistados.

\section{Referências}

1. Cais DP, Turrini RNT, Strabelli TMV. Infecções em pacientes submetidos a procedimento hemodialítico: revisão sistemática. Rev Bras Ter Intensiva. 2009;21(3):269-75. http://dx.doi.org/10.1590/S0103-507X2009000300006.

2. Ministério da Saúde (BR). Portaria n. 2.616, de 12 de maio de 1998. Determina a criação das Coordenações Estaduais e Distrital de Controle de Infecção Hospitalar - CECIH, como executores locais do Programa de Controle de Infecções Hospitalares. Brasília, DF: MS; 1998.

3. Oliveira AC, Paula AO, Iquiapaza RA, Lacerda ACS. Health care associated infections and severity of illness index of patients in intensive care units. Rev Gaucha Enferm. 2012;33(3):89-96. https://doi.org/10.1590/S1983-14472012000300012.

4. Nogueira LS, Ferretti-Rebustini REL, Poveda VB, Silva RCG, Barbosa RL, Oliveira EM, et al. Carga de trabalho de enfermagem: preditor de infecção relacionada à assistência à saúde na terapia intensiva? Rev Esc Enferm USP. 2015;49(n. esp):36-42. https://doi.org/10.1590/S0080623420150000700006 .

5. Ministério da Saúde (BR), Agência Nacional de Vigilância Sanitária. Portaria n. 196, de 24 de junho de 1983. Institui a implantação de Comissões de Controle de Infecção Hospitalar em todos os hospitais do país, independentemente de sua natureza jurídica. Brasília, DF: MS/ANVISA; 1983.

6. Rodrigues PMA, Carmo Neto E, Santos LRC, Knibel MF. Pneumonia associada à ventilação mecânica: epidemiologia e impacto na evolução clínica de pacientes em uma unidade de terapia intensiva. J Bras Pneumol. 2009;35(11):1084-91. https://doi.org/10.1590/S180637132009001100005.

7. Duarte SV, Furtado MS. Manual para elaboração de monografias e projetos de pesquisa. Montes Claros: UNIMONTES; 1999.

8. Ministério da Saúde (BR), Conselho Nacional de Saúde. Resolução n. 196, de 10 de outubro de 1996. Aprovam diretrizes e normas regulamentadoras de pesquisas envolvendo seres humanos. Brasília, DF: MS/CNS; 1996.

9. Albuquerque AM, Souza MA, Torres VSF, Porto VA, Soares MJGO, Torquato IMB. Avaliação e prevenção da úlcera por pressão pelos enfermeiros de terapia intensiva: conhecimento e 
prática. Rev Enferm UFPE on line. 2014;8(2):229-39. 10.5205/1981-8963-v8i2a9666p229-2392014.

10. Afecto MCP, Teixeira MB. Evaluation of occupational stress and burnout syndrome in nurses of an intensive care unit: a qualitative study. Online Braz J Nurs. 2009;8(1):1-12. https://doi.org/10.5935/1676-4285.20092107.

11. Ouriques CM, Machado ME. Enfermagem no processo de esterilização de materiais. Texto Contexto - Enferm. 2013;22(3):695-703. https://doi.org/10.1590/S0104-07072013000300016.

12. UNIMONTES, Hospital Universitário Clemente de Farias, Comissão de Controle de Infecção Hospitalar. Manual de controle de infecção hospitalar. Montes Claros, MG: CCIH/HUCF/UNIMONTES; 2006.

13. Silva SBR, Fonseca JFA. Manual de medidas de prevenção das principais infecções hospitalares. Cuiabá: CCIH/HGCF; 2006.

14. Conterno LO, Toni SMD, Konkiewitz RG, Guedes ES, Barros RT, Tiveron MG. Impact of hospital infections on patients outcomes undergoing cardiac surgery at Santa Casa de Misericórdia de Marília. Rev Bras Cir Cardiovasc. 2014;29(2):167-76. https://doi.org/10.5935/16789741.20140037.

15. Guimarães AC, Donalisio MR, Santiago THR, Freire JB. Óbitos associados à infecção hospitalar, ocorridos em um hospital geral de Sumaré-SP, Brasil. Rev Bras Enferm. 2011;64(5):864-9. https://doi.org/10.1590/S0034-71672011000500010.

16. Silva SG, Nascimento ERP, Salles RK. Pneumonia associada à ventilação mecânica: discursos de profissionais acerca da prevenção. Esc Anna Nery. 2014;18(2):290-5. https://doi.org/10.5935/1414-8145.20140042.

17. Silva QCG, Barbosa MH. Risk factors for surgical site infection in cardiac surgery. Acta Paul Enferm. 2012;25(n. esp 2):89-95. http://dx.doi.org/10.1590/S0103-21002012000900014.

18. Andrade AM, Borges KS, Lima HO. Avaliação das coberturas para sítio de inserção do cateter venoso central no TMO: analise de custos. Rev Min Enferm. 2011;15(2):233-41.

19. Barros LFNM, Arênas VG, Bettencourt ARC, Diccini S, Fram DS, Belasco AGS et al. Avaliação do tipo de curativo utilizado em cateter venoso central para hemodiálise. Acta Paul Enferm. 2009;22(n. esp 1):481-6. https://doi.org/10.1590/S0103-21002009000800004. 
20. Oliveira AC, Cardoso CS, Mascarenhas D. Precauções de contato em unidade de terapia intensiva: fatores facilitadores e dificultadores para adesão dos profissionais. Rev Esc Enferm USP. 2010;44(1):161-5. https://doi.org/10.1590/S0080-62342010000100023.

21. Costa MCP, Taminato M, Silva V, Grande AJ, Beretta ALRZ. A biossegurança durante os cuidados de enfermagem aos pacientes com tuberculose pulmonar. Acta Paul Enferm. 2013;26(4):30712. https://doi.org/10.1590/S0103-21002013000400002.

22. Ferreira AAA, Queiroz KCS, Torres KP, Ferreira MAF, Accioly $H$, Alves MSCF. Os fatores associados à tuberculose pulmonar e a baciloscopia: uma contribuição ao diagnóstico nos serviços de saúde pública. Rev Bras Epidemiol. 2005;8(2):142-9. https://doi.org/10.1590/S1415790X2005000200006.

23. Universidade Federal do Rio de Janeiro, Hospital Universitário Clementino Fraga Filho, Comissão de Controle de Infecção Hospitalar. Infecção hospitalar: medidas de prevenção. Rio de Janeiro: CCIH/HUCFF/UFRJ; 2008.

24. Mesquita APCC. Análise do conhecimento dos graduandos de enfermagem quanto às medidas de precaução e isolamento [monografia]. [São Paulo]: Instituto Hoyler ${ }_{\text {" }} 2006$.

\section{Minicurrículo}

Juliana Aparecida Versiani de Souza | ORCiD: 0000-0001-8326-5335

Acadêmica do curso de graduação em Medicina pela Faculdade de Medicina de Marabá (FACIMPA). Enfermeira. Especialista em Gestão de Programa de Saúde da Família pelas Faculdades Integradas de Jacarepaguá (FIJ). Marabá, PA, Brasil.

Patrick Leonardo Nogueira da Silva | ORCiD: 0000-0003-2399-9526

Acadêmico do curso de graduação em Medicina pelas Faculdades Unidas do Norte de Minas (FUNORTE). Enfermeiro. Especialista em Saúde da Família, Didática e Metodologia do Ensino, Enfermagem do Trabalho. Mestrando do Programa de Pós-Graduação em Cuidado Primário em Saúde (PPGCPS) pela Universidade Estadual de Montes Claros (UNIMONTES). Professor da Escola Técnica de Saúde do Centro de Educação Profissional e Tecnológica da UNIMONTES. Montes Claros, MG, Brasil.

Renata Patrícia Fonseca Gonçalves | ORCiD: 0000-0002-5292-2053

Enfermeira. Especialista em Controle de Infecção Hospitalar e Assistência Cardiovascular. Doutora em Enfermagem pelo Programa de Pós-Graduação em Enfermagem (PPGENF) da Escola de Enfermagem da Universidade Federal de Minas Gerais (EE/UFMG). Professora do Departamento de Enfermagem da Universidade Federal dos Vales do Jequitinhonha e Mucuri (UFVJM). Diamantina, MG, Brasil. 